\title{
GFP-Mutant Human Tau Transgenic Mice Develop Tauopathy Following CNS Injections of Alzheimer's Brain-Derived Pathological Tau or Synthetic Mutant Human Tau Fibrils
}

\author{
(1)Garrett S. Gibbons, ${ }^{\star}$ Rachel A. Banks, ${ }^{\star}$ Bumjin Kim, Hong Xu, Lakshmi Changolkar, Susan N. Leight, \\ Dawn M. Riddle, Chi Li, Ronald J. Gathagan, Hannah J. Brown, Bin Zhang, John Q. Trojanowski, \\ and $\odot$ Virginia M.-Y. Lee \\ Department of Pathology and Laboratory Medicine and Center for Neurodegenerative Disease Research, Perelman School of Medicine at the University of \\ Pennsylvania, Philadelphia, Pennsylvania 19104
}

\begin{abstract}
Neurodegenerative proteinopathies characterized by intracellular aggregates of tau proteins, termed tauopathies, include Alzheimer's disease (AD), frontotemporal lobar degeneration (FTLD) with tau pathology (FTLD-tau), and related disorders. Pathological tau proteins derived from human AD brains (AD-tau) act as proteopathic seeds that initiate the templated aggregation of soluble tau upon intracerebral injection into tau transgenic ( $\mathrm{Tg}$ ) and wild-type mice, thereby modeling human tau pathology. In this study, we found that aged $\mathrm{Tg}$ mice of both sexes expressing human tau proteins harboring a pathogenic P301L MAPT mutation labeled with green fluorescent protein (T40PL-GFP Tg mouse line) exhibited hyperphosphorylated tau mislocalized to the somatodentritic domain of neurons, but these mice did not develop de novo insoluble tau aggregates, which are characteristic of human $\mathrm{AD}$ and related tauopathies. However, intracerebral injections of either T40PL preformed fibrils (PFFs) or AD-tau seeds into T40PL-GFP mice induced abundant intraneuronal pathological inclusions of hyperphosphorylated T40PL-GFP. These injections of pathological tau resulted in the propagation of tau pathology from the injection site to neuroanatomically connected brain regions, and these tau inclusions consisted of both T40PL-GFP and WT endogenous mouse tau. Primary neurons cultured from the brains of neonatal T40PL-GFP mice provided an informative in vitro model for examining the uptake and localization of tau PFFs. These findings demonstrate the seeded aggregation of T40PL-GFP in vivo by synthetic PFFs and human AD-tau and the utility of this system to study the neuropathological spread of tau aggregates.
\end{abstract}

Key words: Alzheimer's disease; tau; tauopathy; transgenic model

Significance Statement

The stereotypical spread of pathological tau protein aggregates have recently been attributed to the transmission of proteopathic seeds. Despite the extensive use of transgenic mouse models to investigate the propagation of tau pathology in vivo, details of the aggregation process such as the early seeding events leading to new tau pathology have remained elusive. This study validates the use of GFP-labeled tau expressed by neurons in vivo and in vitro as models for investigating mechanisms underlying the seeded transmission of tau pathology as well as tau-focused drug discovery to identify disease-modifying therapies for AD and related tauopathies.

\section{Introduction}

Intracellular inclusions formed by pathological tau proteins are the signature lesions of a class of neurodegenerative diseases termed tauopathies, including Alzheimer's disease (AD) and frontotemporal lobar degeneration (FTLD) with tau pathology (FTLD-tau; Lee et al., 2001). The natively unstructured microtubule-binding protein
Received Aug. 23, 2017; revised Sept. 25, 2017; accepted Sept. 28, 2017.

Author contributions: G.S.G., R.A.B., S.N.L., C.L., and V.M.-Y.L. designed research; G.S.G., R.A.B., B.K., H.X., L.C., S.N.L., D.M.R., C.L., R.J.G., H.J.B., and B.Z. performed research; G.S.G., L.C., D.M.R., and V.M.-Y.L. contributed unpublished reagents/analytic tools; G.S.G., R.A.B., B.K., J.Q.T., and V.M.-Y.L. analyzed data; G.S.G., R.A.B., and V.M.-Y.L. wrote the paper.

This work was supported by National Institutes of Health Grants AG053036 (G.S.G.), AG10124 (J.Q.T.), and AG17586 (V.M.-Y.L.); and the Wyncote Foundation. We thank John Robinson, Theresa Schuck, and Katie Casalnova for assistance with obtaining suitable brains for these studies. We also thank Peter Davies for his generous gift of the PHF1 antibody.

${ }^{*}$ G.S.G. and R.A.B. contributed equally to this work.

The authors declare no competing financial interests.

Correspondence should be addressed to Virginia M.-Y. Lee, 3rd floor Maloney Building, 3600 Spruce Street, Philadelphia, PA 19104.E-mail: vmylee@upenn.edu.

DOI:10.1523/JNEUROSCI.2393-17.2017

Copyright $\odot 2017$ the authors $\quad 0270-6474 / 17 / 3711485-10 \$ 15.00 / 0$ 
tau is primarily expressed in CNS neurons as six alternatively spliced isoforms generated by the MAPT gene on chromosome 17, and they contain $0-2 \mathrm{~N}$-terminal acidic regions $(0-2 \mathrm{~N}$ tau isoforms) and three or four microtubule-binding domain repeats (3R or $4 \mathrm{R}$ tau isoforms; Ballatore et al., 2007). In tauopathies, tau proteins are converted into diverse species of insoluble aggregates as exemplified by neurofibrillary tangles (NFTs) in AD (Dickson et al., 2011; Irwin et al., 2015). Furthermore, $>30$ pathogenic MAPT mutations have been identified in families with hereditary frontotemporal dementia with parkinsonism linked to chromosome 17 (FTDP-17), also referred to as familial FTLD-Tau (Hutton et al., 1998; Lee et al., 2001; Spillantini and Goedert, 2013). The MAPT exon 10 mutation that converts proline 301 to leucine (P301L) decreases the microtubule binding and increases the aggregation of the corresponding mutant $4 \mathrm{R}$ tau isoforms (Hong et al., 1998; Hutton et al., 1998; Nacharaju et al., 1999). Similarly, the MAPT mutation leading to the substitution of proline at position 301 to serine (P301S) causes an early-onset, rapidly progressive form of FTDP-17 in combination with epileptic seizures (Bugiani et al., 1999; Sperfeld et al., 1999).

Notably, transgenic ( $\mathrm{Tg}$ ) mice expressing tau proteins with a P301L or P301S mutation develop AD-like tau tangles and are useful models for investigating mechanisms of disease in $\mathrm{AD}$ and related tauopathies (Lewis et al., 2000; Allen et al., 2002; Yoshiyama et al., 2007). Expression of P301L tau (0N4R) in the CNS driven by the mouse prion promoter results in AD-like NFT pathology at 4.5 months of age in the JNPL3 Tg tauopathy mouse model (Lewis et al., 2000). Similarly, 2N4R tau containing the P301L mutation expressed from the Thy1.2 promoter, leads to the accumulation of hyperphosphorylated tau that is aberrantly localized from axons to the somatodendritic compartment of neurons in the pR5 murine model (Götz et al., 2001). PS19 mice containing the P301S mutation in human tau (1N4R) were generated using the mouse prion promoter driving expression of mutant human tau at approximately fivefold higher levels than endogenous mouse tau in the CNS (Yoshiyama et al., 2007). Pathological tau extracted from human tauopathy brains or synthetic tau preformed fibrils (PFFs) generated in vitro seed aggregation of mutant human tau in PS19 mice upon intracerebral injection, thereby providing strong evidence for the transmission of pathological tau in vivo (Iba et al., 2013; Boluda et al., 2015).

A $\mathrm{Tg}$ mouse model expressing green fluorescent protein (GFP)-labeled tau could provide a means to monitor the temporal sequence, spatial distribution, and dose-dependent templated fibrillization process induced by CNS injections of pathological tau in vivo, as well as providing a useful in vitro model using neurons cultured from the brains of these Tg mice. Notably, a Tg mouse expressing human $\alpha$-synuclein fused to GFP enabled studies of $\alpha$-synuclein aggregation processes in vivo over time using live imaging (Spinelli et al., 2014). However, it is not clear whether GFP-labeled tau protein would exhibit aggregation properties in vivo following CNS injections of $\mathrm{AD}$-brain-derived tau (ADtau) or synthetic tau PFFs similar to that observed with unlabeled tau proteins expressed by tau Tg mouse models. To address these issues, we established a Tg mouse line expressing the 2N4R human tau T40 isoform with the P301L mutation tagged with GFP (T40PL-GFP) at the $\mathrm{C}$ terminus in the CNS driven by the mouse prion promoter using methods similar to those described (Yoshiyama et al., 2007).

Most attempts to exploit GFP-tagged tau to analyze tau dynamics have used viral transductions to express GFP-tagged tau in vivo or in vitro. While these models have been useful for investigating aspects of tau aggregation and transmission, the variability in expression due to transient transfection makes these models less reliable than stable expression of a tau-GFP construct. To study the process of neuronal tau aggregation in vivo, we used intracerebral injections of tau fibrils purified from AD brains (AD-tau) and synthetic tau PFFs into the CNS of our new T40PLGFP mouse model as well as neurons cultured from the brains of these Tg mice that were exposed to AD-tau and tau PFFs.

\section{Materials and Methods}

T40PL-GFP Tg mice. Site-directed mutagenesis was used to convert amino acid 301 of the T40 tau isoform (2N4R) from proline to leucine (P301L), and this mutation was confirmed by sequencing followed by ligation to GFP. The cDNA construct of the P301L mutant human T40 tau with GFP was cloned into the MoPrP.Xho expression vector at the XhoI site downstream of the mouse prion promoter (Borchelt et al., 1996). T40PL-GFP Tg mice were created on a B6C3/F1 background using the $15.5 \mathrm{~kb}$ NotI fragment containing T40-GFP. Stable heterozygous $\left(\mathrm{T}_{40 \mathrm{PL}-\mathrm{GFP}^{+/-}}\right.$) and homozygous (T40PL-GFP ${ }^{+/+}$) Tg lines carrying the T40PL-GFP transgene were established. Both male and female mice were used for this study, and no differences between sexes were observed in our analyses. All animal care and experimental protocols were approved by the Institutional Animal Care and Use Committee at the University of Pennsylvania.

Fluorescence microscopy. Mice were anesthetized with intraperitoneal injection of ketamine/xylazine then transcardially perfused with $30 \mathrm{ml}$ of PBS at $120 \mathrm{ml} / \mathrm{h}$. For frozen sectioning, brains were removed and fixed in $4 \%$ paraformaldehyde (PFA) for $24 \mathrm{~h}$ at $4^{\circ} \mathrm{C}$ followed by sucrose saturation at $4^{\circ} \mathrm{C}$ for $24 \mathrm{~h}$ and several PBS washes. Brains were subsequently frozen in optimal cutting temperature compound on dry ice. Frozen brains were cut into $20-\mu \mathrm{m}$-thick sections, and fluorescent images acquired on a Leica DMI6000 microscope and a PerkinElmer Lamina slide scanner.

Sequential extraction of mouse brains. Frozen brain tissue from mice of both sexes was weighed and sonicated in $9 \times$ tissue weight high-salt/RAB buffer, containing $100 \mathrm{~mm}$ 2-[ $\mathrm{N}$-morpholino] ethanesulfonic acid, $1 \mathrm{mM}$ EGTA, $0.5 \mathrm{~mm} \mathrm{MgSO}_{4}, 0.75 \mathrm{M} \mathrm{NaCl}$, and $20 \mathrm{~mm} \mathrm{NaF}$ supplemented with protease and phosphatase inhibitors as well as phenylmethylsulfonyl fluoride and centrifuged at $100,000 \times g$ for $30 \mathrm{~min}$ at $4^{\circ} \mathrm{C}$. Supernatants were analyzed as soluble fractions, and the pellets were resuspended by sonication in $9 \times$ volume of high-salt/RAB/1\% Triton $\mathrm{X}-100$ buffer and centrifuged at $100,000 \times g$ for $30 \mathrm{~min}$ at $4^{\circ} \mathrm{C}$. The pellets were resuspended by sonication in PHF buffer containing $10 \mathrm{~mm}$ Tris, $\mathrm{pH}$ 7.6, 0.85 M NaCl, 1 mm EDTA, $20 \mathrm{~mm} \mathrm{NaF,} \mathrm{10 \%} \mathrm{sucrose,} \mathrm{and} \mathrm{1 \%} \mathrm{Sarkosyl} \mathrm{fol-}$ lowed by rotation at room temperature for $1 \mathrm{~h}$ and then centrifuged at $100,000 \times g$ for $30 \mathrm{~min}$ at $4^{\circ} \mathrm{C}$. Supernatants were removed, and the pellets were washed with PBS and centrifuged at $100,000 \times g$ for $30 \mathrm{~min}$ at $4^{\circ} \mathrm{C}$ then resuspended in PBS to generate the final insoluble fractions. Protein concentrations in each fraction were determined by BCA assay, and $15 \mu \mathrm{g}$ of total protein was run on a 7.5\% SDS-PAGE gel. The insoluble fractions were resuspended in PBS and loaded at a fourfold greater amount than the soluble fractions. Protein was transferred to $0.2 \mu \mathrm{m}$ nitrocellulose membrane, blocked with $5 \%$ nonfat milk in Tris-buffered saline with $0.1 \%$ Triton X-100 for $30 \mathrm{~min}$ at room temperature, then immunoblotted with primary antibodies diluted in $5 \%$ nonfat milk in TBS at dilutions described in Table 1 for $1 \mathrm{~h}$ at room temperature. The membrane was washed and probed with infrared dye-conjugated secondary antibodies (1:20,000 dilution) in 5\% nonfat milk. Membranes were scanned using an Odyssey Imaging System (LI-COR).

Statistical analysis. Total soluble tau extracted from aging T40PL$\mathrm{GFP}^{+/+}$was measured by immunoblot as described above. Quantification of T40PL-GFP protein was determined for three mice each at 6, 9, and 12 months of age, using Image Soft software and normalized to GAPDH protein levels. Statistical analysis was performed using GraphPad Prism software by one-way ANOVA with Tukey's post hoc test.

Tau PFF generation. T40PL PFFs were generated by mixing $40 \mu \mathrm{M}$ purified recombinant T40PL tau with $40 \mu \mathrm{m}$ heparin and $2 \mathrm{~mm}$ DTT in PBS followed by shaking at $37^{\circ} \mathrm{C}$ for $5 \mathrm{~d}$. Before injection in the brains of mice, the mixture was centrifuged at $100,000 \times g$ for $30 \mathrm{~min}$ at $22^{\circ} \mathrm{C}$ and the pellet was resuspended in PBS. Fluorophore-tagged T40PL-594 PFFs 
Table 1. Antibodies used in the study including Research Resource Identifiers where available

\begin{tabular}{|c|c|c|c|c|}
\hline Antibody name & Specificity & Host species & Dilutions & Source (reference or RRID) \\
\hline K9JA & Human and mouse tau C-terminal (amino acids 243-441) & Rabbit polyclonal & $1: 5000(\mathrm{WB})$ & Dako (RRID:AB_10013724) \\
\hline T49 & Mouse tau & Mouse monoclonal & 1:1000 (ICC) & In-house (Kosik et al., 1988) \\
\hline R2295M & Mouse tau & Rabbit polyclonal & $1: 1000$ (IF) & In-house (Guo et al., 2016) \\
\hline DMR180 & Human tau N-terminal & Mouse monoclonal & $1: 1000$ (IF) & Newly generated in-house \\
\hline PHF-1 & p-tau (phosphorylated at Ser396 and Ser404) & Mouse monoclonal & $1: 5000$ (WB) & Gift from Dr. Peter Davies (Greenberg et al., 1992) \\
\hline AT8 & p-tau (phosphorylated at Ser202 and Thr 205) & Mouse monoclonal & 1:10,000 (IHC), 1:5000 (IF) & Thermo Fisher Scientific (RRID:AB_223647) \\
\hline NFL1/2 & Neurofilament light chain & Rabbit polyclonal & $1: 500$ (ICC) & In-house (Balin and Lee, 1991) \\
\hline GFP & Green fluorescent protein & Mouse monoclonal & 1:1000 (IHC) & Santa Cruz Biotechnology (RRID:AB_627695) \\
\hline GAPDH (6C5) & Glyceraldehyde-3-phosphate dehydrogenase & Mouse monoclonal & $1: 5000$ (WB) & Advanced Immunochemical (NA) \\
\hline MAP2 (17028) & Microtubule-associated protein & Rabbit polyclonal & $1: 500$ (ICC) & In-house (Volpicelli-Daley et al., 2011) \\
\hline
\end{tabular}

ICC, Immunocytochemistry; RRID, Research Resource Identifier; WB, Western blot.

were generated by labeling recombinant monomeric T40PL using a Alexa Fluor 594 microscale protein labeling kit (Life Technologies) to generate T40PL-594. Fibrillization reactions were prepared with $2 \mu \mathrm{M}$ T40PL-594 and $38 \mu \mathrm{M}$ T40PL monomer to generate PFFs containing 5\% labeled T40PL. AD-tau seeded T40PL PFFs (T40PL AD-P1) were prepared as previously described (Guo et al., 2016). Briefly, $4 \mu \mathrm{M}$ AD-tau was incubated with $36 \mu \mathrm{M}$ T40PL monomer in PBS and shaken at $37^{\circ} \mathrm{C}$ for $3 \mathrm{~d}$. Fibrillization was monitored by sedimentation assay using ultracentrifugation and analysis by SDS-PAGE gels followed by Coomassie Blue staining of the soluble and pelleted fractions.

Stereotaxic intracerebral injections. Two- to 3-month-old T40PL-GFP or $\mathrm{B} 6 \mathrm{C} 3 / \mathrm{F} 1$ mice of both sexes were deeply anesthetized with ketamine/ xylazine/acepromazine and immobilized in a stereotaxic frame (David Kopf Instruments) installed with both a stereotaxic robot and a microinjection robot (Neurostar) for motorized, computer-controlled injections. Animals were aseptically injected with synthetic tau PFFs, AD-tau, or PBS in the dorsal hippocampus unilaterally $(-2.5 \mathrm{~mm}$ from bregma; lateral, $+2 \mathrm{~mm}$ from bregma; depth, $-2.4 \mathrm{~mm}$ from the skull) using a Hamilton syringe. The injection site received $2.5 \mu \mathrm{l}$ of tau proteins using both the synthetic tau PFFs and AD-tau, which were sonicated before injection at a concentration of $0.8 \mu \mathrm{g} / \mu \mathrm{l}(2 \mu \mathrm{g} / \mathrm{site})$.

Antibodies used in this study. Table 1 summarizes the antibodies used in this study.

Immunohistochemistry. Paraffin-embedded PFA fixed brains were sectioned at $6 \mu \mathrm{m}$. Following deparrafinization in xylene and rehydration, endogenous peroxidases were inactivated by treatment with methanol/ hydrogen peroxide for $30 \mathrm{~min}$ at room temperature. Tissue was blocked with $2 \%$ fetal bovine serum in $0.1 \mathrm{~m}$ Tris, $\mathrm{pH} 7.6$, and stained with primary antibodies diluted in blocking buffer at $4^{\circ} \mathrm{C}$ overnight. Antibody binding was detected with a chromogenic horseradish peroxidase development solution (Biogenex) and counterstained with hematoxylin.

Heatmaps of tau pathology. Coronal tissue sections of varying bregma depths spanning the entire brain were stained for AT8 by immunohistochemistry (IHC), as described above. Tissue sections spanning the hippocampus (distance from bregma: $-1.22,-2.18,-2.92,-3.52$, and $-4.48 \mathrm{~mm}$ ) were graded by a semiquantitative pathological tau score for each region and averaged for three mice per group. Semiquantitative scores of tau pathology were mapped onto the brain regions as previously described (Iba et al., 2013).

Primary neuronal culture. Primary hippocampal neurons were dissected from embryonic day 16 (E16) to E18 T40PL-GFP ${ }^{+/+}$mice and plated on poly-D-lysine-coated coverslips. Cells were grown in Neurobasal media with $1 \%$ GlutaMAX, $2 \%$ B27, and $1 \%$ penicillin/streptomycin at $37^{\circ} \mathrm{C}$ with $5 \% \mathrm{CO}_{2}$, as described previously (Guo and Lee, 2013).

PFF uptake and tau aggregation in primary neuron cultures. Primary hippocampal neuron generation from T40PL-GFP ${ }^{+/+}$mice as described above were used at DIV $7-10$ and treated with $1 \mu \mathrm{g}$ of sonicated T40PL PFFs or T40PL-594 PFFs per well of a 24-well plate. Live cell imaging was performed $3 \mathrm{~h}$ after treatment, and a $z$-stack of images was obtained throughout the focal plane for cell bodies. Alternatively, live cell imaging was obtained over time, and cells were maintained in an imaging chamber at $37^{\circ} \mathrm{C}$ with $5 \% \mathrm{CO}_{2}$. For tau aggregation, cells were incubated for $14 \mathrm{~d}$ after treatment with media exchanged $7 \mathrm{~d}$ after treatment. For chloroquine treatment, media were removed $3 \mathrm{~h}$ after treatment with T40PL-
GFP PFFs and replaced with media containing $200 \mu \mathrm{M}$ chloroquine for $30 \mathrm{~min} 37^{\circ} \mathrm{C}$. Coverslips were removed from the 24 -well plate, washed with PBS, and transferred to a new 24-well plate containing $500 \mu \mathrm{l}$ of conditioned neuronal media per well and incubated for $7 \mathrm{~d}$. Cells were fixed with 4\% PFA and 10\% sucrose in PBS, and imaged on a Leica DMI6000 Microscope.

\section{Results}

T40PL-GFP localizes to somatodendritic compartment of neurons in T40PL-GFP Tg mice

Since P301L tau isoforms show diminished binding to microtubules and increased self-assembly into filamentous insoluble aggregates (Hong et al., 1998), we generated Tg mice that express a GFP-conjugated form of the longest human tau isoform, T40, containing a P301L mutation (T40PL-GFP) driven by the mouse prion promoter. Initial characterization of the transgene expression level was examined by immunoblot (IB) of cortical extracts from 2 - to 3-month-old T40PL-GFP ${ }^{+/+}$homozygous and T40PL-GFP ${ }^{+/-}$heterozygous mice compared with agematched WT and PS19 mice. IB with the pan-tau rabbit polyclonal antibody K9JA with similar affinity for human and mouse tau showed human T40PL-GFP expression that was approximately equivalent to endogenous levels of mouse tau expression. The total expression of tau in T40PL-GFP mice was approximately twofold higher than in WT mice and was comparable to the expression levels of tau in the PS19 Tg mice (Fig. 1A). Although initially reported to have human tau expression fivefold greater than endogenous tau, there has been an apparent downward shift in the expression of tau in the PS19 line over time, resulting in slightly decreased human tau expression relative to endogenous mouse tau (Yoshiyama et al., 2007).

To define the regional distribution of T40PL-GFP expression, we examined GFP signal by fluorescence microscopy in $20-\mu \mathrm{m}$ thick frozen brain sections. T40PL-GFP was expressed throughout the brain with particularly high expression in the cortex and hippocampus and low expression in the cerebellum, olfactory bulbs, and brainstem (Fig. 1B). The accumulation of T40PL-GFP was observed in cell bodies of the dentate gyrus granular cell layer and within axonal projections into the molecular layer in 6-month-old mice (Fig. 1C). Subcellular localization of T40PLGFP was determined using double immunofluorescence (IF) examination of T40PL-GFP primary neurons cultured in vitro. T40PL-GFP expression was observed mainly in neuronal perikarya, whereas endogenous mouse tau was primarily localized to axons (Fig. 2). Furthermore, T40PL-GFP colabeled the dendritic compartment of cells stained by the microtubule-associated protein 2 (MAP2) and partially with the axonal marker neurofilament light chain (NFL 1/2; Fig. 2). Redistribution from axons to the somatodendritic compartment was attributed to the overexpression of the $\mathrm{P} 301 \mathrm{~L}$ protein and the effect of this mutation on 
A

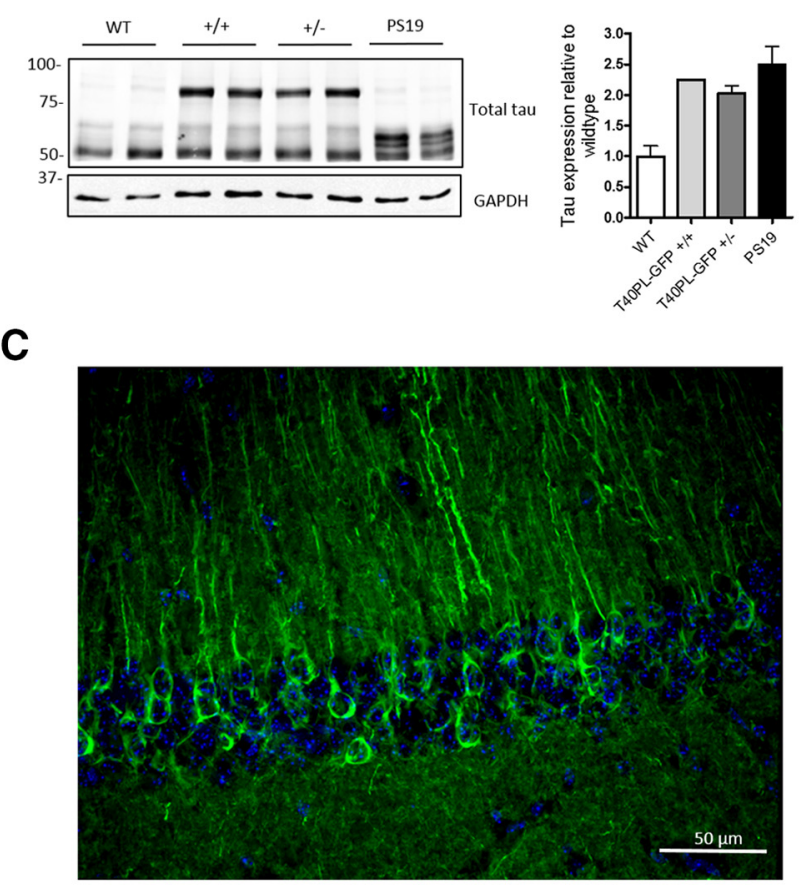

B
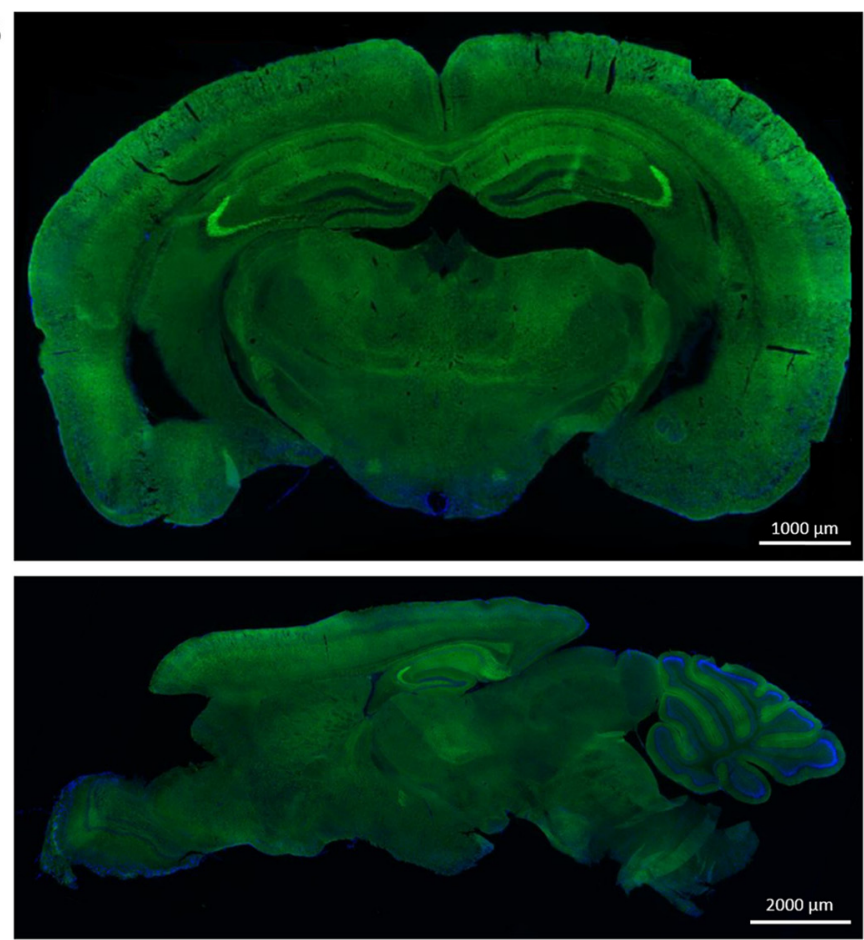

Figure 1. GFP-labeled mutant human tau overexpression results in somatodendritic mislocalization of T4OPL-GFP. A, Immunoblot analysis with a rabbit polyclonal K9JA antibody that detects total tau extracted from cortices of 2- to 3-month-old WT mice and T4OPL-GFP as well as PS19 tau Tg mice. The endogenous mouse tau band migrates at 55 kDa, while the T40PL-GFP tau band migrates at $75 \mathrm{kDa}$. Immunoblot quantification of total tau protein in transgenic mouse models relative to WT mice. $\boldsymbol{B}$, Fluorescent signal of T40PL-GFP and DAPI in coronal and sagittal $20-\mu \mathrm{m}$-thick sections from frozen brain tissue sections from 3-month-old T40PL-GFP homozygous mice. C, 3D deconvolution of a $z$-stack of fluorescent images showing T40PL-GFP expression and DAPI in the dentate gyrus and hippocampus CA1 in a 20- $\mu$ m-thick section of frozen brain tissue from homozygous T40PL-GFP Tg mouse.

its reduced binding to microtubules, as described earlier (Yoshiyama et al., 2007; Helboe et al., 2017).

\section{T40PL-GFP Tg mice do not develop de} novo tau pathology with advancing age To examine whether the overexpression of T40PL-GFP results in hyperphosphorylated insoluble tau aggregates, we performed sequential extraction of hippocampal tissue from T40PL-GFP and PS19 mice at various ages. We observed that nearly all of the overexpressed T40PLGFP protein is extracted in the initial soluble fraction of CNS proteins despite the presence of T40PL-GFP that is phosphorylated at the Ser 396/404 epitope indicated by PHF1 staining (Greenberg et al., 1992; Fig. $3 A$ ). These results also demonstrate a greater overexpression of T40PL-GFP relative to endogenous mouse tau in the hippocampus compared with the cortical extracts. There were no age-dependent changes in the levels of T40PL-GFP protein expression (Fig. 3B; one-way ANOVA, $p=0.133)$, Ser 396/404 phosphorylation, or solubility in T40PL-GFP mice up to 12 month of age. As previously reported, PS19 mice developed increases in the phosphorylation of tau and accumulation in the insoluble fraction with ad-
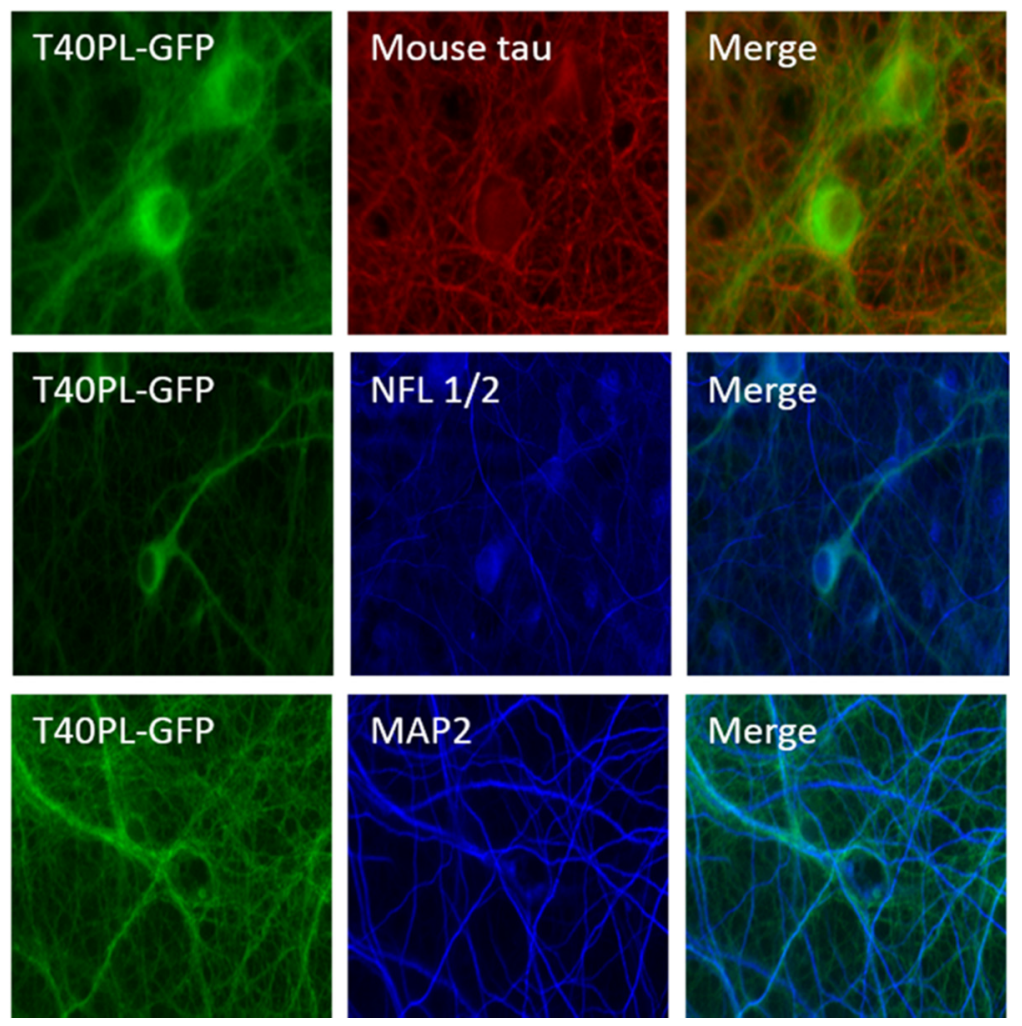

Figure 2. Subcellular localization of T40PL-GFP in primary neurons. Coimmunofluoresence of T40PL-GFP primary neurons $14 \mathrm{~d}$ in vitro fixed with 4\% paraformaldehyde and stained for mouse tau (T49); NFL 1/2, which serves as an axonal marker; and MAP2, a marker of the somatodendritic compartment. 
A

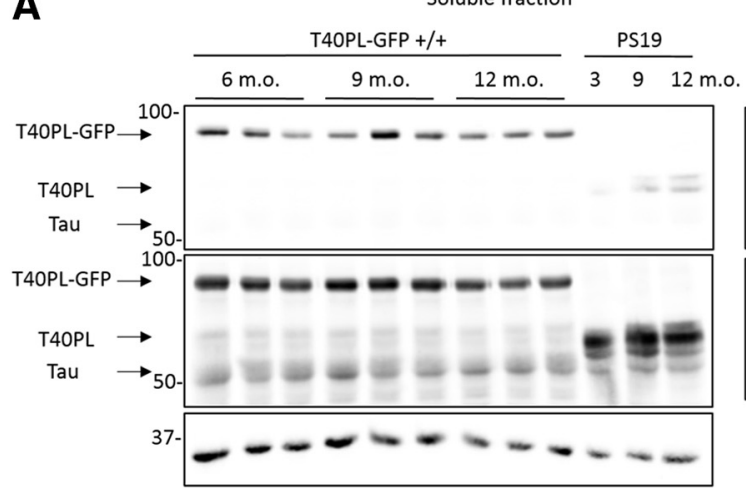

Insoluble fraction

\begin{tabular}{|c|c|c|c|c|c|}
\hline \multicolumn{3}{|c|}{ T40PL-GFP +/+ } & \multicolumn{3}{|c|}{ PS19 } \\
\hline 6 m.o. & 9 m.o. & 12 m.o. & 3 & 9 & $12 \mathrm{~m} . \mathrm{o}$ \\
\hline
\end{tabular}

C

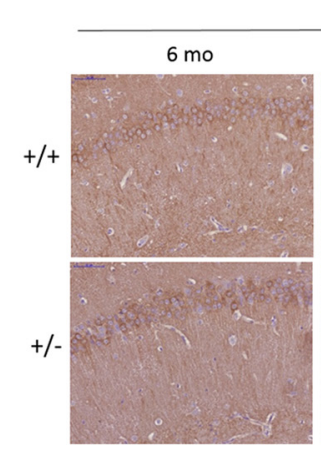

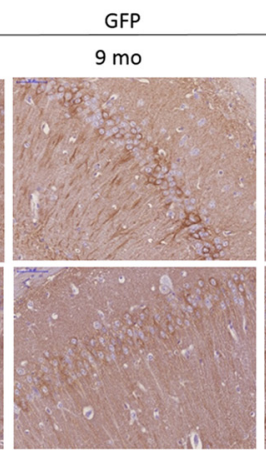
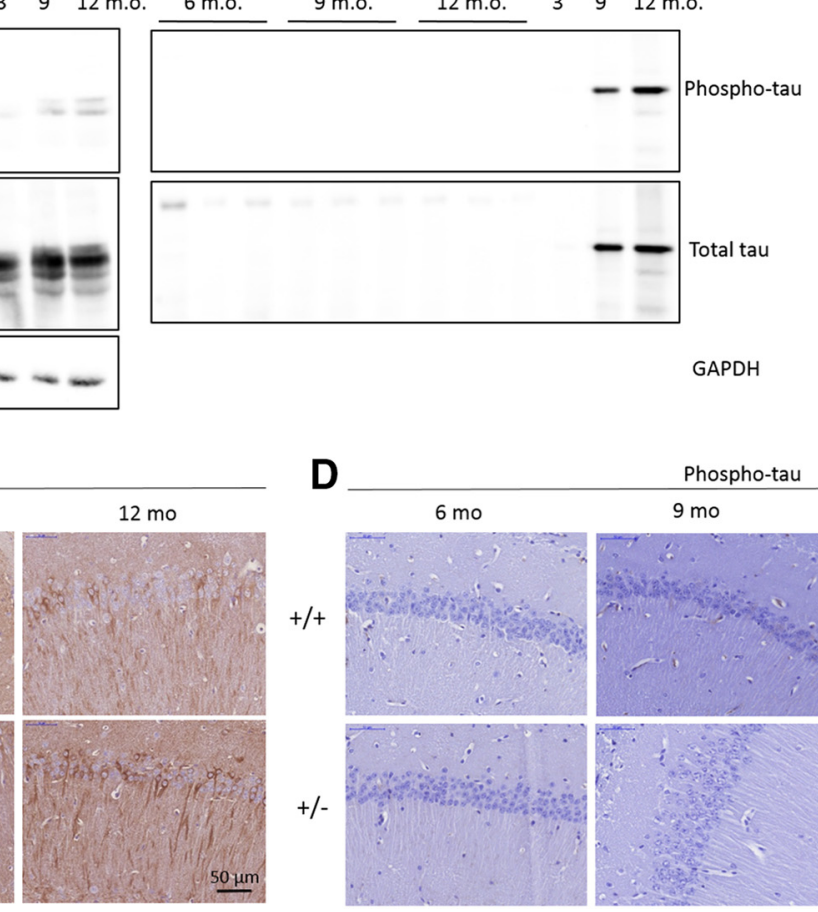

GAPDH

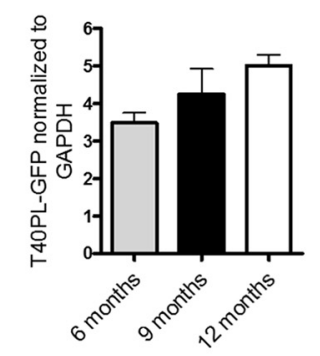

B

Figure 3. Aging T40PL-GFP mice do not develop de novo insoluble tau aggregates. $\boldsymbol{A}$, Western blot analysis of soluble and insoluble tau extracts from hippocampi of aged T40PL-GFP and PS19 Tg mice. Phospho-tau immunoblotting was performed using PHF1 antibody, which recognizes phosphorylated tau at Ser 396/404. Total tau immunoblotting was performed with the polyclonal pan-tau antibody K9JA, and GAPDH was used as a loading control. The soluble fraction consists of protein extracted by high-salt RAB buffer followed by sequential extraction with $1 \%$ Triton X-100 and then $1 \%$ Sarkosyl. The insoluble fraction consists of the Sarkosyl-insoluble proteins resuspended and sonicated in PBS. B, Immunoblot quantification of soluble T40PL-GFP relative to GAPDH reveals no statistically significant change in T40PL-GFP protein levels with age. One-way ANOVA with Tukey's post hoc test was performed: $p=0.133$. $C$, IHC reveals the presence of GFP protein and demonstrates the high expression levels of the T40PL-GFP protein in the hippocampus of aged T40PL-GFP homozygous (+ /+) and heterozygous (+ / - ) mice. D, IHC with AT8, which recognizes tau phosphorylated at residues S202 and T205, is completely negative in the T40PL-GFP mice, demonstrating that the hyperphosphorylated tau detected by biochemical methods does not form insoluble aggregates in vivo.

vancing age. Despite the high expression of T40PL-GFP and its mislocalization to the somatodendritic compartment, as shown by IHC with anti-GFP antibodies (Fig. $3 C$ ), there was a lack of AT8 (phospho Ser202/Thr205) staining by IHC (Fig. 3D), which is a signature of pathological tau. These results indicate that although T40PL-GFP accumulates in cell bodies and is phosphorylated at Ser 396/404, it remains soluble and does not mature into AT8-positive intraneuronal AD-like NFTs in the T40PLGFP Tg mice up to 12 months of age.

\section{T40PL-GFP aggregates are seeded in vivo by intracerebral injections of synthetic tau PFFs and AD-tau}

To determine whether recombinant human tau (T40PL) PFFs or human $\mathrm{AD}$-tau is capable of seeding the aggregation of T40PLGFP in vivo, we performed unilateral injections of T40PL PFFs or $\mathrm{AD}$-tau into the hippocampus of 2- to 3-month-old T40PL-GFP mice and examined their brains for evidence of tau pathology 3 months postinjection (p.i.) by IHC with AT8 (Fig. 4). We observed that both $\mathrm{AD}$-tau and T40PL PFFs induced tau aggregates in T40PL-GFP ${ }^{+/+}$and T40PL-GFP ${ }^{+/-}$Tg mice. Synthetic T40PL PFFs did not induce tau pathology in WT mice 3 months p.i., as expected from our previous studies (Guo et al., 2016). T40PL PFF injections produced fewer intracellular tau aggregates in T40PL$\mathrm{GFP}^{+/-}$mice compared with T40PL-GFP ${ }^{+/+}$mice. Neither PBS-injected nor age-matched uninjected Tg T40PL-GFP mice developed any AT8-positive tau pathology (data not shown), indicating that damage from injections does not induce nonspecific tau pathology and that the tau pathology described above was due to seeding by the injected pathological tau rather than by de novo aggregation of the tau proteins expressed by the transgene.

T40PL-GFP $^{+/+}$and T40PL-GFP ${ }^{+/-}$mice had comparable burdens of tau pathology after injection of $\mathrm{AD}$-tau or T40PL PFFs, although there was somewhat less tau pathology induced by CNS injections of AD-tau than by T40PL PFFs (Fig. 4). This finding demonstrates that AT8 signal is not due to the detection of injected tau material as T40PL PFFs are not phosphorylated at Ser202/Thr205 in agreement with the previous observation that injected AD-tau material is not detectable $7 \mathrm{~d}$ postinjection (Guo et al., 2016). WT mice developed AT8-positive neuronal tau pathology primarily in the caudal hippocampus following CNS injections of AD-tau, which was not observed upon injection of T40PL PFFs (Fig. 4). Together, these findings indicate a preferential seeding of T40PL-GFP by T40PL PFFs compared with ADtau, whereas in WT mice, endogenous mouse tau is preferentially seeded by AD-tau compared with T40PL PFFs.

To investigate the extent of the templated spread or transmission of pathological tau in the brains of T40PL-GFP mice induced by T40PL PFFs or AD-tau, every 30th section from serial sections of the entire brain were stained by IHC to detect AT8-positive tau pathology, which was semiquantitatively mapped onto coronal brain sections to generate CNS heatmaps of this pathology (Fig. 5). Tau pathology was most abundant in CA3, subiculum, retrosplenial granular cortex, and the polymorph layer of the dentate gyrus after CNS injections of T40PL PFFs. AD-tau CNS injections 

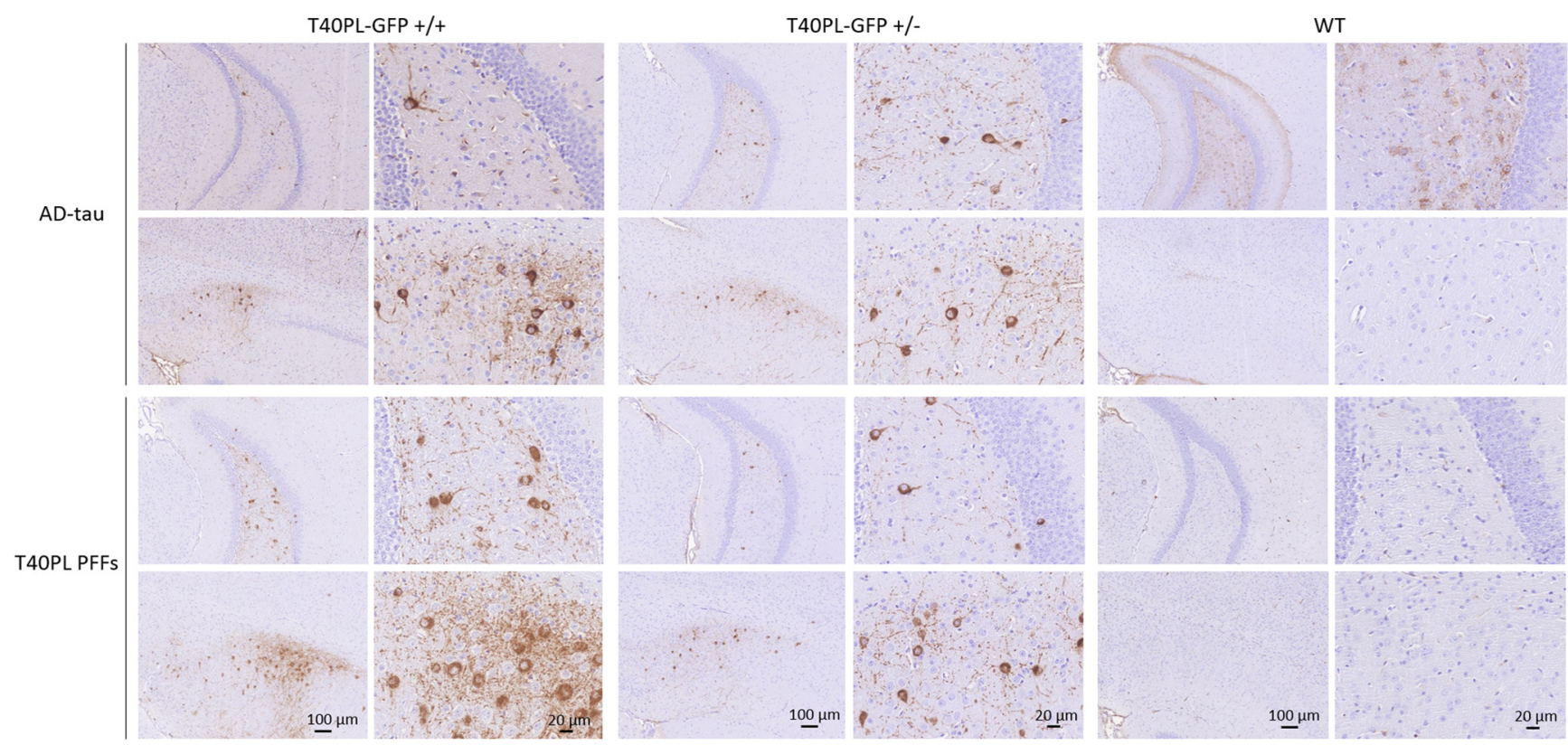

Figure 4. AD-tau and T4OPL PFFs seed tau aggregation in T4OPL-GFP Tg mice. Analysis of tau pathology induced by AD-tau or in vitro fibrillized T40PL PFFs injected into the dorsal hippocampus of 3-month-old T40PL-GFP homozygous or heterozygous T4OPL-GFP Tg mice or WT non-Tg control mice assessed 3 months p.i. by IHC with AT8. Representative images of caudal hippocampus and subiculum are shown with $n \geq 3$ mice/group.

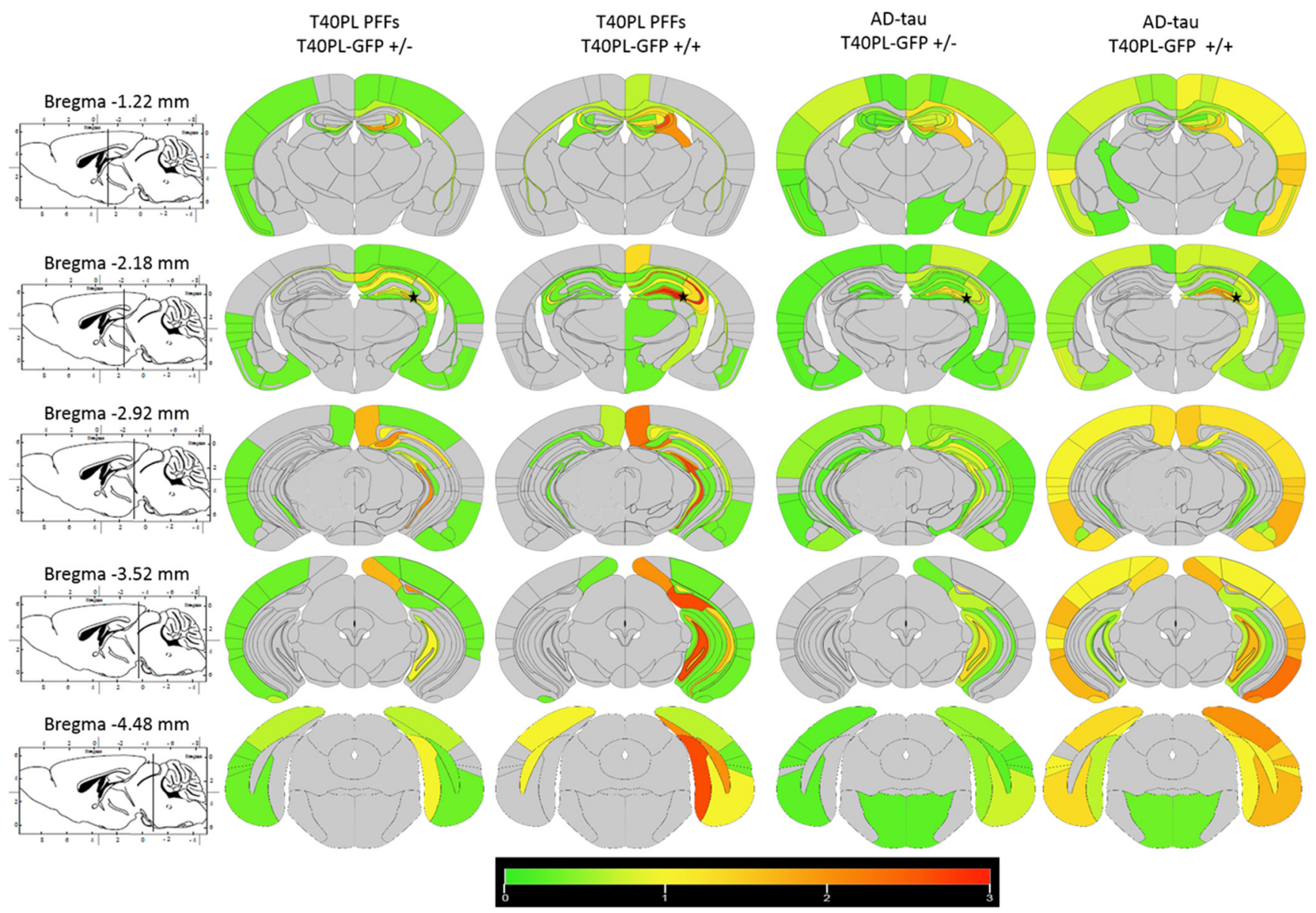

Figure 5. Propagation of tau pathology in T4OPL-GFP mice following intracerebral injections of T40PL-PFFs and AD-tau. Heatmaps of AT8-positive tau immunostaining in 6-month-old T4OPL-GFP homozygous or heterozygous mice 3 months p.i. with either AD-tau or T40PL-PFFs. Coronal sections represent rostral (top) to caudal coronal planes, which are indicated in the left panels. The black star indicates the injection site. 


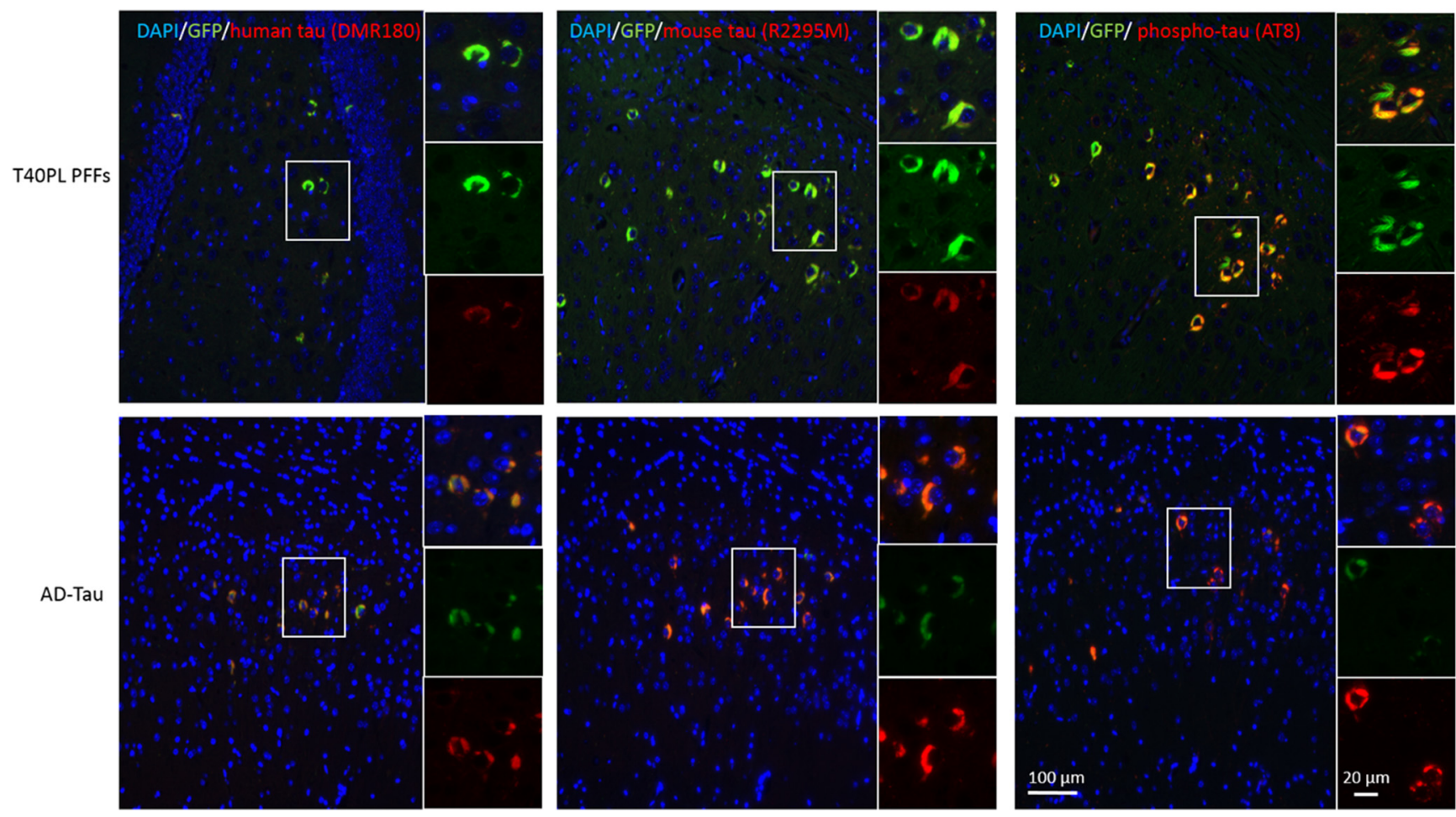

Figure 6. Mouse tau and human T40PL-GFP coaggregate into hyperphosphorylated tau inclusions seeded by T40PL PFFs or AD-tau. IF staining of GFP and human tau-specific antibody DMR180 confirm that there is no cleavage of the GFP tag. Colocalization of GFP and mouse tau-specific antibody R2295M or AT8 demonstrate coaggregation of mouse tau and human T40PL-GFP into AD-like tangle pathology.

seeded the development of tau pathology in the same areas, but there was more cortical tau pathology, especially in the entorhinal cortex. While the majority of the propagated tau pathology was on the ipsilateral side of the injections, there was some spread to the contralateral hemisphere after injections of PFFs or AD-tau. T40PL-GFP ${ }^{+/+}$mice developed greater contralateral tau pathology than T40PL-GFP ${ }^{+/-}$mice, but the patterns of the spread of tau pathology were similar on the ipsilateral and contralateral hemispheres. Consistent with our prior findings in the PS19 model (Iba et al., 2013; Boluda et al., 2015), overexpression of P301S mutant human tau facilitates the spread of tau pathology after seeding with injected tau PFFs or AD-tau. Yet, neither ADtau or T40PL PFFs induced tau aggregates in the spinal cords of T40PL-GFP homozygous or heterozygous mice (data not shown). Interestingly, the burden of tau pathology did not directly correlate with T40PL-GFP expression levels, indicating that tau pathology spread is influenced by neuroanatomical connections and cell type-selective vulnerability to a greater extent than the transgene regulated expression levels of tau in the T40PL-GFP model.

Tau aggregates seeded by injecting AD-tau and T40PL-GFP PFFs into the brains of T40PL-GFP Tg mice consist of both T40PL-GFP and endogenous mouse tau

Next, we sought to determine whether tau aggregates induced by AD-tau and T40PL PFFs in T40PL-GFP mice consist of T40PLGFP, endogenous mouse tau, or both by using double IF (Fig. 6). In both T40PL PFF- and AD-tau-injected T40PL-GFP mice, all GFP-positive aggregates detected by green IF of the expressed T40PL-GFP were also immunostained by the human tau-specific antibody DMR180. This suggests that the T40PL-GFP fusion protein is intact and that there are no proteolytic cleavage fragments of GFP alone forming aggregates independent of tau protein. This observation is further supported by the biochemical extraction of full-length T40PL-GFP fusion protein from aged uninjected T40PL-GFP mice shown above. Interestingly, T40PLGFP aggregates also costained for mouse tau with the mouse tau-specific polyclonal antibody R2295M, which was immunodepleted of human tau-reactive IgG. This finding demonstrates that T40PL-GFP inclusions coaggregate with endogenous mouse tau in addition to the overexpressed mutant human tau. Last, GFP aggregates colocalized with AT8 indicating that the pathological aggregates are hyperphosphorylated, consistent with previous IHC findings. Thus, in T40PL-GFP-expressing Tg mice, T40PL-GFP forms phosphorylated pathological tau aggregates that also recruit endogenous mouse tau in vivo after seeding by CNS injections of either T40PL PFFs or AD-tau.

Synthetic tau PFFs are internalized and seed tau aggregates in cultured T40PF-GFP Tg mouse primary neurons

To investigate whether primary neurons generated from the hippocampus of T40PL-GFP Tg mice are useful for probing cellular mechanisms of tau PFF uptake and trafficking, we performed fluorescence microscopy, including live cell-imaging experiments with Alexa Fluor 594-labeled T40PL fibrils (T40PL-594). T40PL-594 PFFs were generated as described in Material and Methods by incorporating 5\% of labeled T40PL-594 with 95\% unlabeled T40PL tau monomers, and the biological activities of these tau PFFs were tested in primary neurons from Tg mice, as described previously (Guo et al., 2016). Low label incorporation (5\%) enabled rapid visualization of internalized T40PL-594 PFFs with minimal signal from external fibrils (Fig. 7A). Upon internalization, T40PL-594 PFFs are accumulated into bright intracellular punctae, while external T40PL-594 PFFs remain diffuse. Fluorescent image acquisition parameters that enable the detection of high-intensity intracellular T40PL-594 PFF punctae require short exposure times that are insufficient to detect the 

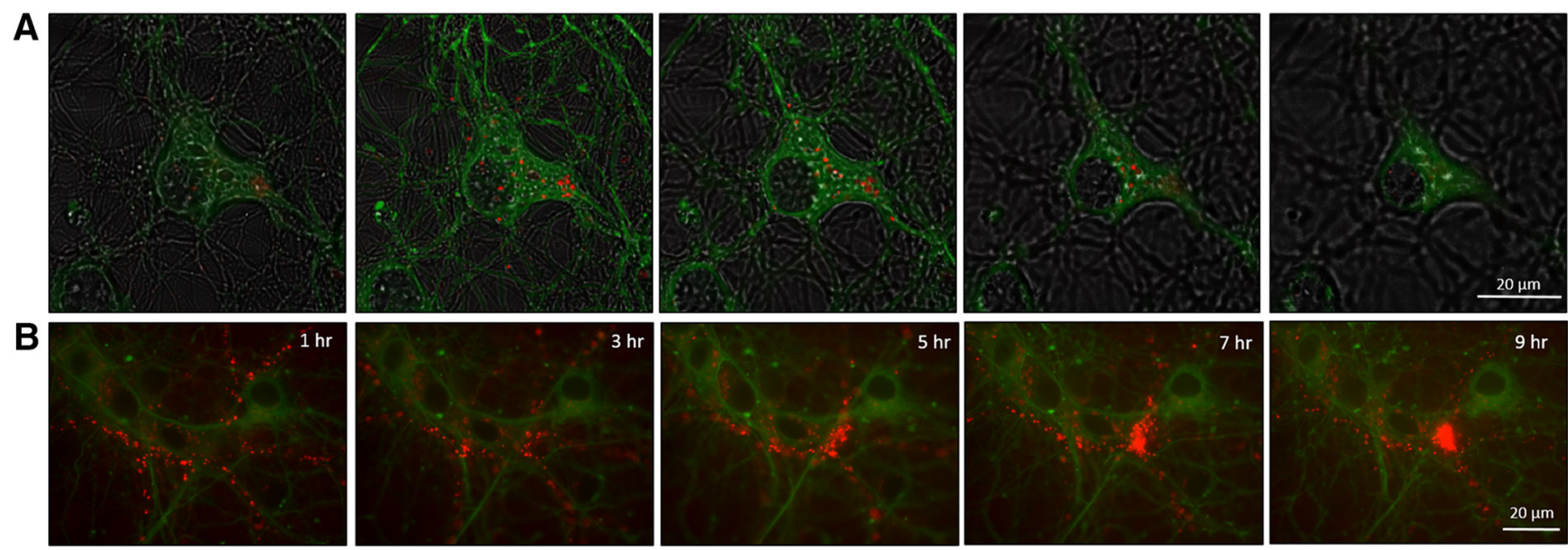

C Chloroquine

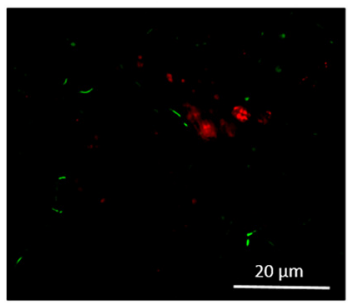

D

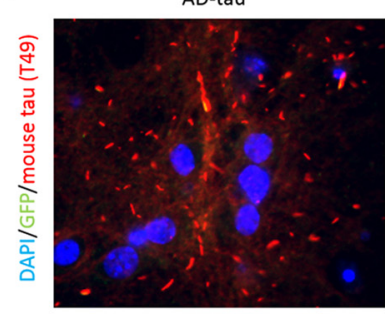

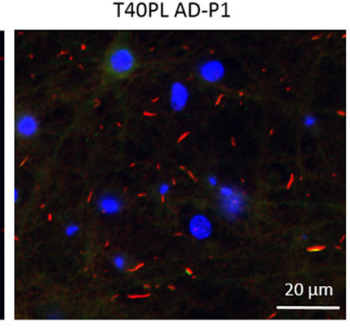

Figure 7. T40PL PFFs are internalized by primary neurons derived from T40PL-GFP Tg mice, and they preferentially seed mouse tau aggregates. $\boldsymbol{A}$, $z$-Stack fluorescent images of T40PL-GFP primary neurons treated with Alexa Fluor 594-labeled T40PL tau incorporated into PFFs (T40PL-594) at low label incorporation (5\%). Image acquisition parameters were optimized for the detection of bright intracellular accumulations and constrasted such that individual external T40PL-594 PFFs are below the detection threshold. $\boldsymbol{B}$, Time course of T40PL-GFP neuronal uptake of T40PL-594 PFFs demonstrating accumulation into cell bodies. C, Treatment of T40PL-GFP-expressing neurons with chloroquine after the application of T40PL-594 PFFs decreases the degradation of PFFs and increases T40PL-GFP aggregation $7 \mathrm{~d}$ after treatment. $D$, Treatment of T40PL-GFP expressing neurons with either AD-tau or AD-seeded recombinant T40PL PFFs (T40PL AD-P1) preferentially induces the aggregation of endogenous mouse tau with limited T40PL-GFP incorporation into the mouse tau aggregates $14 \mathrm{~d}$ after treatment. $n=3$ independent biological replicates.

low-intensity signal from dispersed extracellular T40PL-594 PFFs. Z-stack images acquired at varying intraneuronal depths revealed perikaryal and neuritic T40PL-594-positive PFF aggregates within the T40PL-GFP-positive cell bodies of cultured Tg mouse primary neurons. Over the time course of $9 \mathrm{~h}$ after treatment of neurons with T40PL-594 PFFs, intracellular punctae were dynamically trafficked within these cultured neurons (Fig. 7B). Initially small and dispersed T40PL-594 punctae were translocated to cell bodies and coalesced into large, bright intracellular accumulations. Thus, these T40PL-GFP Tg primary neurons provide a valuable tool to rapidly assess the internalization of T40PL-594 fibrils without the need for extracellular fluorescence quenching reagents or trypsinization, and this model system also enabled temporal resolution of dynamic tau aggregate trafficking processes within neurons.

To test the hypothesis that exogenously added T40PL-594 PFFs are processed through the lysosomal/endosomal pathway upon uptake by neurons, we evaluated the effects of chloroquine on T40PL-GFP aggregation in neurons generated from T40PL$\mathrm{GFP}^{+/+}$mice. Chloroquine is a highly basic small molecule that impairs lysosomal function by altering the $\mathrm{pH}$ gradient across lysosomal membranes (Wibo and Poole, 1974). Cultured T40PLGFP homozygous Tg primary neurons were treated with T40PL594 PFFs, and the recruitment of T40PL-GFP protein into fibrils was assessed $7 \mathrm{~d}$ after treatment. We observed that more T40PL594 PFFs were retained in the chloroquine-treated cells than in untreated cells, which is consistent with the chloroquine-induced inhibition of lysosomal PFF degradation (Fig. 7C). Additionally, chloroquine treatment increased the number of T40PL-GFP aggregates, suggesting that the inhibition of T40PL PFF seed degra- dation leads to enhanced recruitment of T40PL-GFP. However, it is not clear whether this is a direct result of increasing T40PL PFF seeds by inhibiting their degradation or whether chloroquine treatment perturbs the lysosomal degradation pathway in a manner that promotes PFF escape from lysosomal compartments, thus increasing contact between T40PL PFF seeds and transgeneexpressed T40PL-GFP substrate for templated aggregation. Nonetheless, these results highlight the utility of this model for cell biological studies to interrogate the kinetics and mechanisms of the seeded aggregation process in neurons.

Finally, to test whether tau aggregates in primary neuron culture models recapitulate those observed in vivo, we cultured E14E16 hippocampal neurons from T40PL-GFP ${ }^{+/+}$mice and treated them with $\mathrm{AD}$-tau and T40PL PFFs seeded by $10 \% \mathrm{AD}$-tau in vitro (T40PL AD-P1). Cultured T40PL-GFP neurons developed tau aggregates $14 \mathrm{~d}$ after treatment with $\mathrm{AD}$-tau and T40PL AD-P1 fibrils (Fig. 7D). In each of these experiments, endogenous mouse tau showed extensive insoluble accumulations, while there were fewer GFP-positive accumulations. Since all the GFP aggregates also were positive for mouse tau (Fig. $7 D$ ), we infer that the mouse tau is preferentially seeded by the exogenously added fibrils and then seeds the accumulation of T40PL-GFP into the aggregate. Thus, the fact that seeded aggregates in T40PL-GFP mouse brain were composed of both T40PL-GFP and endogenous mouse tau (Fig. 6) suggests that T40PL-GFP can be recruited to and colocalize with nearly all mouse tau aggregates with time.

\section{Discussion}

We have shown that a Tg mouse line (T40PL-GFP) expressing human full-length mutant human tau fused with GFP is capable 
of forming intracellular hyperphosphorylated tau aggregates upon intracerebral injections of synthetic mutant tau PFFs or pathological AD-tau that act as proteopathic seeds to induce templated propagation of both endogenous mouse tau and the transgenically expressed T40PL-GFP. In these mice, T40PL-GFP is highly expressed in the hippocampus and cortex, where it is mislocalized to the somatodendritic compartment. This mislocalization is consistent with reports that T40PL diminishes binding to microtubules, leading to its mislocalization compared with WT tau (Nacharaju et al., 1999). However, the somatodendritic mislocalization of T40PL-GFP alone did not lead to the formation of AD-like tau pathology, since the uninjected mice living up to 12 months of age did not form AT8-positive tau inclusions. Furthermore, sequential biochemical extraction revealed that T40PL-GFP was phosphorylated at the Ser 396/404 epitope, yet it remained soluble and did not convert to an insoluble pathological species of tau as the mice age up to 12 months, as is evident by the absence of AT8 IHC staining of tissue. While the lack of de novo tau pathology may have previously limited the applications of this model system, using the pathological tau seeded aggregation paradigm allowed us generate a compelling GFP-labeled model of an AD-like human tauopathy.

T40PL-GFP mice formed pathological tau aggregates following intracerebral injection with either AD-tau or synthetic T40PL PFFs. Homozygous mice developed greater tau pathology than heterozygous mice when seeded with T40PL-PFFs, whereas ADtau induced similar burdens of tau pathology. In the case of the homozygous T40PL-GFP ${ }^{+/+}$Tg mice, high T40PL-GFP expression provides abundant substrate to propagate T40PL PFFs and less T40PL-GFP substrate is available in the heterozygous T40PL$\mathrm{GFP}^{+/-}$mice. However, in both cases, there are equivalent levels of endogenous mouse tau, and the development of similar levels of tau pathology seeded by $\mathrm{AD}$-tau suggests that endogenous mouse tau may be the initially recruited species of tau that is propagated by AD-tau. This is supported by the following additional lines of evidence: (1) although mouse tau is present in T40PL PFF-induced aggregates at 3 months p.i., there is greater endogenous mouse tau colocalized with T40PL-GFP in AD-tau induced inclusions at this time; and (2) when we examined seeding events in primary neurons, mouse tau was more readily seeded by AD-tau with minimal T40PL-GFP coaggregation at these early time points. Together, these findings suggest that the GFP tag on tau may provide a modest kinetic barrier for nucleation with $\mathrm{AD}$-tau but, given sufficient time in vivo, T40PL-GFP is recruited into pathological tau aggregates seeded by either T40PL PFFs or AD-tau.

The spreading pattern of pathological tau in T40PL-GFP mice is similar to other Tg mutant human tau mouse models following intracerebral injection with tau PFF seeds (Iba et al., 2013). Injection of K18/T40 PFFs into the hippocampus of PS19 mice resulted in tau pathology throughout the hippocampus and entorhinal cortex at 1 month postinjection, similar to the observations for T40PL-GFP mice injected with T40PL fibrils at 3 months postinjection. Furthermore, the injection of T40PL-GFP mice with $\mathrm{AD}$-tau resulted in more abundant cortical pathology than those injected with T40PL PFFs; this is in agreement with injections of extracts from a Down's syndrome patient with AD pathology in the PS19 Tg mice (Boluda et al., 2015). However, there was more ipsilateral to contralateral spread of tau pathology in the PS19 mice than in the T40PL-GFP mice after the injection of either tau PFFs or AD-tau. This may be due to slowed kinetics of tau aggregate formation resulting from the GFP tag, which resulted in a slower transneuronal spread of pathology. Overall, the pattern of spread of pathology in the T40PL-GFP mice was consistent with what has been previously described for PS19 mice and with a model of transynaptic transmission of pathological tau (Liu et al., 2012).

Culturing of primary neurons derived from T40PL-GFP with stable and consistent tau expression levels that can be visualized provides a compelling in vitro model system in which to investigate the dynamics of seeded tau aggregation without the need for viral infection. Live imaging of this model will allow interrogation of the cell biology of cell-to-cell transmission, including the uptake and trafficking of exogenously added tau PFFs and ADtau, as well as studies of the aggregation of T40PL-GFP over time. Time-lapse microscopy revealed the accumulation of fluorescently labeled synthetic T40PL-594 PFFs internalized by neurons occurring over several hours. To observe tau PFF internalization over time, we adopted a strategy of low T40PL-594 incorporation into PFFs, allowing for relatively dim extracellular fibrils to be contrasted below the detection threshold. The resulting intracellular accumulations of the punctate T40-594 PFF signal represent the most abundant accumulations of PFFs and not necessarily individual PFFs. Fluorescence quenching methods recently applied by our laboratory use high label incorporation into PFFs for more sensitive detection of individual internalized species (Karpowicz et al., 2017). To investigate the fate of internalized tau PFFs and AD-tau, we used chloroquine, a small molecule that disrupts lysosomal function (Gonzalez-Noriega et al., 1980; Chen et al., 2011). Treatment of neurons with chloroquine resulted in decreased degradation of exogenously added PFFs and increased accumulation of T40PL-GFP aggregates, suggesting that the inhibition of the lysosomal degradation of tau PFFs increased the speed with which T40PL-GFP aggregated, as they were not observed at the same time point after treatment in the absence of chloroquine. Accordingly, by increasing the amount of tau PFF seeds through blocking their degradation, more nucleation sites are available for the recruitment of T40PL-GFP into aggregates. These data further support the notion that tau PFFs taken up in neurons are trafficked for degradation via the lysosomal pathway as was demonstrated for $\alpha$-synuclein PFFs in WT mouse primary neurons (Karpowicz et al., 2017). Disruption of this pathway therefore accelerates the tau aggregation process and increases the amount of tau pathology seeded by the tau PFFs. Thus, this new in vitro model system provided new insights into the process of seeded aggregation of tau in cultured neurons.

\section{References}

Allen B, Ingram E, Takao M, Smith MJ, Jakes R, Virdee K, Yoshida H, Holzer M, Craxton M, Emson PC, Atzori C, Migheli A, Crowther RA, Ghetti B, Spillantini MG, Goedert M (2002) Abundant tau filaments and nonapoptotic neurodegeneration in transgenic mice expressing human P301S tau protein. J Neurosci 22:9340-9351. Medline

Balin BJ, Lee VM (1991) Individual neurofilament subunits reassembled in vitro exhibit unique biochemical, morphological and immunological properties. Brain Res 556:196-208. CrossRef Medline

Ballatore C, Lee VM, Trojanowski JQ (2007) Tau-mediated neurodegeneration in Alzheimer's disease and related disorders. Nat Rev Neurosci 8:663-672. CrossRef Medline

Boluda S, Iba M, Zhang B, Raible KM, Lee VM, Trojanowski JQ (2015) Differential induction and spread of tau pathology in young PS19 tau transgenic mice following intracerebral injections of pathological tau from Alzheimer's disease or corticobasal degeneration brains. Acta Neuropathol 129:221-237. CrossRef Medline

Borchelt DR, Davis J, Fischer M, Lee MK, Slunt HH, Ratovitsky T, Regard J, Copeland NG, Jenkins NA, Sisodia SS, Price DL (1996) A vector for expressing foreign genes in the brains and hearts of transgenic mice. Genet Anal 13:159-163. CrossRef Medline

Bugiani O, Murrell JR, Giaccone G, Hasegawa M, Ghigo G, Tabaton M, 
Morbin M, Primavera A, Carella F, Solaro C, Grisoli M, Savoiardo M, Spillantini MG, Tagliavini F, Goedert M, Ghetti B (1999) Frontotemporal dementia and corticobasal degeneration in a family with a P301S mutation in tau. J Neuropathol Exp Neurol 58:667-677. CrossRef Medline

Chen PM, Gombart ZJ, Chen JW (2011) Chloroquine treatment of ARPE-19 cells leads to lysosome dilation and intracellular lipid accumulation: possible implications of lysosomal dysfunction in macular degeneration. Cell Biosci 1:10. CrossRef Medline

Dickson DW, Kouri N, Murray ME, Josephs KA (2011) Neuropathology of frontotemporal lobar degeneration-tau (FTLD-tau). J Mol Neurosci 45: 384-389. CrossRef Medline

Gonzalez-Noriega A, Grubb JH, Talkad V, Sly WS (1980) Chloroquine inhibits lysosomal enzyme pinocytosis and enhances lysosomal enzyme secretion by impairing receptor recycling. J Cell Biol 85:839-852. CrossRef Medline

Götz J, Chen F, Barmettler R, Nitsch RM (2001) Tau filament formation in transgenic mice expressing P301L tau. J Biol Chem 276:529-534. CrossRef Medline

Greenberg SG, Davies P, Schein JD, Binder LI (1992) Hydrofluoric acidtreated tau PHF proteins display the same biochemical properties as normal tau. J Biol Chem 267:564-569. Medline

Guo JL, Lee VM (2013) Neurofibrillary tangle-like tau pathology induced by synthetic tau fibrils in primary neurons over-expressing mutant tau. FEBS Lett 587:717-723. CrossRef Medline

Guo JL, Narasimhan S, Changolkar L, He Z, Stieber A, Zhang B, Gathagan RJ, Iba M, McBride JD, Trojanowski JQ, Lee VM (2016) Unique pathological tau conformers from Alzheimer's brains transmit tau pathology in nontransgenic mice. J Exp Med 213:2635-2654. CrossRef Medline

Helboe L, Egebjerg J, Barkholt P, Volbracht C (2017) Early depletion of CA1 neurons and late neurodegeneration in a mouse tauopathy model. Brain Res 1665:22-35. CrossRef Medline

Hong M, Zhukareva V, Vogelsberg-Ragaglia V, Wszolek Z, Reed L, Miller BI, Geschwind DH, Bird TD, McKeel D, Goate A, Morris JC, Wilhelmsen KC, Schellenberg GD, Trojanowski JQ, Lee VM (1998) Mutation-specific functional impairments in distinct tau isoforms of hereditary FTDP-17. Science 282:1914-1917. CrossRef Medline

Hutton M, Lendon CL, Rizzu P, Baker M, Froelich S, Houlden H, PickeringBrown S, Chakraverty S, Isaacs A, Grover A, Hackett J, Adamson J, Lincoln S, Dickson D, Davies P, Petersen RC, Stevens M, de Graaff E, Wauters E, van Baren J, Hillebrand M, Joosse M, Kwon JM, Nowotny P, Che LK, Norton J, Morris JC, Reed LA, Trojanowski J, Basun H, Lannfelt L, Neystat M, Fahn S, Dark F, Tannenberg T, Dodd PR, Hayward N, Kwok JB, Schofield PR, Andreadis A, Snowden J, Craufurd D, Neary D, Owen F, Oostra BA, Hardy J, Goate A, van Swieten J, Mann D, Lynch T, Heutink P (1998) Association of missense and $5^{\prime}$-splice-site mutations in tau with the inherited dementia FTDP-17. Nature 393:702-705. CrossRef Medline

Iba M, Guo JL, McBride JD, Zhang B, Trojanowski JQ, Lee VM (2013) Synthetic tau fibrils mediate transmission of neurofibrillary tangles in a trans- genic mouse model of Alzheimer's-like tauopathy. J Neurosci 33:10241037. CrossRef Medline

Irwin DJ, Cairns NJ, Grossman M, McMillan CT, Lee EB, Van Deerlin VM, Lee VM, Trojanowski JQ (2015) Frontotemporal lobar degeneration: defining phenotypic diversity through personalized medicine. Acta Neuropathol 129:469-491. CrossRef Medline

Karpowicz RJ Jr, Haney CM, Mihaila TS, Sandler RM, Petersson EJ, Lee VM (2017) Selective imaging of internalized proteopathic alpha-synuclein seeds in primary neurons reveals mechanistic insight into transmission of synucleinopathies. J Biol Chem 292:13482-13497. CrossRef Medline

Kosik KS, Orecchio LD, Binder L, Trojanowski JQ, Lee VM, Lee G (1988) Epitopes that span the tau-molecule are shared with paired helical filaments. Neuron 1:817-825. CrossRef Medline

Lee VM, Goedert M, Trojanowski JQ (2001) Neurodegenerative tauopathies. Annu Rev Neurosci 24:1121-1159. CrossRef Medline

Lewis J, McGowan E, Rockwood J, Melrose H, Nacharaju P, Van Slegtenhorst M, Gwinn-Hardy K, Paul Murphy M, Baker M, Yu X, Duff K, Hardy J, Corral A, Lin WL, Yen SH, Dickson DW, Davies P, Hutton M (2000) Neurofibrillary tangles, amyotrophy and progressive motor disturbance in mice expressing mutant (P301L) tau protein. Nat Genet 25:402-405. CrossRef Medline

Liu L, Drouet V, Wu JW, Witter MP, Small SA, Clelland C, Duff K (2012) Trans-synaptic spread of tau pathology in vivo. PLoS One 7:e31302. CrossRef Medline

Nacharaju P, Lewis J, Easson C, Yen S, Hackett J, Hutton M, Yen SH (1999) Accelerated filament formation from tau protein with specific FTDP-17 missense mutations. FEBS Lett 447:195-199. CrossRef Medline

Sperfeld AD, Collatz MB, Baier H, Palmbach M, Storch A, Schwarz J, Tatsch K, Reske S, Joosse M, Heutink P, Ludolph AC (1999) FTDP-17: an earlyonset phenotype with parkinsonism and epileptic seizures caused by a novel mutation. Ann Neurol 46:708-715. CrossRef Medline

Spillantini MG, Goedert M (2013) Tau pathology and neurodegeneration. Lancet Neurol 12:609-622. CrossRef Medline

Spinelli KJ, Taylor JK, Osterberg VR, Churchill MJ, Pollock E, Moore C, Meshul CK, Unni VK (2014) Presynaptic alpha-synuclein aggregation in a mouse model of Parkinson's disease. J Neurosci 34:2037-2050. CrossRef Medline

Volpicelli-Daley LA, Luk KC, Patel TP, Tanik SA, Riddle DM, Stieber A, Meaney DF, Trojanowski JQ, Lee VM (2011) Exogenous alpha-synuclein fibrils induce Lewy body pathology leading to synaptic dysfunction and neuron death. Neuron 72:57-71. CrossRef Medline

Wibo M, Poole B (1974) Protein degradation in cultured cells. II. The uptake of chloroquine by rat fibroblasts and the inhibition of cellular protein degradation and cathepsin B1. J Cell Biol 63:430-440. CrossRef Medline

Yoshiyama Y, Higuchi M, Zhang B, Huang SM, Iwata N, Saido TC, Maeda J, Suhara T, Trojanowski JQ, Lee VM (2007) Synapse loss and microglial activation precede tangles in a P301S tauopathy mouse model. Neuron 53:337-351. CrossRef Medline 